\title{
Venous thromboembolism in emergency general surgery patients: a single-centre retrospective cohort study
}

\author{
Mei Yang, MD \\ Patrick B. Murphy, MD \\ Laura Allen, MSc \\ Nathalie Sela, MD \\ Shaylan Govind, MD \\ Ken Leslie, MD \\ Kelly Vogt, MD
}

Poster presentation at the 2016 Canadian Surgery Forum, Toronto, Ontario, Sept. 9, 2016

Accepted June 11, 2019

\section{Correspondence to:}

K. Vogt

London Health Sciences Centre

800 Commissioners Rd East

London ON N6A 5W9

Kelly.Vogt@Ihsc.on.ca

DOI: $10.1503 /$ cjs.006318
Background: There is limited literature on the risk of venous thromboembolism (VTE) in emergency general surgery (EGS) patients. We undertook this study to identify the rate of symptomatic VTE for patients undergoing EGS operations.

Methods: We conducted a retrospective cohort study evaluating EGS patients who underwent operative intervention between March and December 2014. Data collected included patient demographics, type of procedure, risk of VTE, VTE prophylaxis, development of symptomatic VTE, and mortality.

Results: We included 767 patients in our analysis. The mean age was $53 \pm 19.7$ years, and $52.2 \%$ of patients were female. Eighteen patients $(2.3 \%)$ experienced VTE in hospital and $12(1.6 \%)$ experienced VTE after discharge. Only $66 \%$ of patients received appropriate VTE prophylaxis. High-risk patients had a higher VTE rate $(7.4 \%$ v. $2.3 \%, p<0.001)$ and higher mortality $(17.6 \%$ v. $4.0 \%, p<0.001)$ than lowto moderate-risk patients.

Conclusion: The risk of VTE in patients requiring EGS is significant and persists after hospital discharge. Further studies on quality improvement with VTE prophylaxis are warranted.

Contexte : La littérature sur le risque de thromboembolie veineuse (TEV) chez les patients soumis à une chirurgie générale urgente est limitée. Nous avons entrepris cette étude afin de mesurer le taux de TEV symptomatique chez les patients ayant subi une intervention urgente en chirurgie générale.

Méthodes : Nous avons procédé à une étude de cohorte rétrospective sur les patients qui ont subi une chirurgie générale urgente entre mars et décembre 2014. Parmi les données recueillies, mentionnons données démographiques, type d'intervention, risque de TEV, thromboprophylaxie, apparition d'une TEV symptomatique et mortalité.

Résultats : Nous avons inclus 767 patients dans notre analyse. L'âge moyen était de $53 \pm 19,7$ ans et $52,2 \%$ des patients étaient de sexe féminin. Dix-huit patients $(2,3 \%)$ ont présenté une TEV en cours d'hospitalisation et $12(1,6 \%)$ après leur congé. Seulement $66 \%$ des patients ont reçu une thromboprophylaxie adéquate. Les patients à haut risque ont présenté des taux de TEV $(7,4 \%$ c. $2,3 \%, p<0,001)$ et de mortalité $(17,6 \%$ c. $4,0 \%, p<0,001)$ plus élevés que les patients présentant un risque faible à modéré.

Conclusion : Le risque de TEV chez les patients soumis à une chirurgie générale urgente est significatif et persiste après le congé hospitalier. Il faudra mener des études plus approfondies sur l'amélioration de la qualité de la thromboprophylaxie.

T he reported incidence of venous thromboembolism (VTE) has been on the rise. There is significant morbidity and mortality associated with the development of VTE, including prolonged hospital admission, risk of bleeding with therapeutic anticoagulation, recurrent disease and reduced survival. ${ }^{1}$ Hospitalized surgical patients are at higher risk of VTE because of immobility and hypercoagulable state; VTE occurs in up to $25 \%$ of these patients. ${ }^{1}$ The incidence of VTE has been studied extensively in specific 
patient populations such as cancer, trauma and orthopedic patients; however, there is little known on the incidence of VTE in emergency general surgery (EGS) patients, a subgroup of patients who undergo surgery on an emergency, rather than elective, basis for an acute illness. Many centres use currently available guidelines for all surgical patients to guide risk assessment and decisions regarding VTE prophylaxis around the time of EGS. These guidelines - created for elective surgical patients - have unknown utility among EGS patients.

Because the risk of VTE has not been studied in the EGS population, there is a clear knowledge gap with respect to both risk and prophylaxis in the EGS population. The purpose of the present study was to determine the rates of symptomatic VTE in EGS patients, both in hospital and 3 months after discharge; whether VTE rates differ based on patient level of risk; and whether compliance with VTE prophylaxis affects VTE rates. The information gained from this study can act as a starting point by contributing data on VTEs in an unstudied patient population.

\section{Methods}

We performed a retrospective cohort study evaluating all patients who required operation for EGS conditions and were admitted to the acute care surgery (ACS) service at London Health Sciences Centre (LHSC) between Mar. 1, 2014, and Dec. 31, 2014. London Health Sciences Centre is a tertiary care referral centre in London, Ontario, with a catchment of 1.5 million people. Patients were identified for the study cohort using the LHSC Operating Room Database, and all patients who received an EGS procedure during the study period were included. Only patients admitted directly from the emergency department to the ACS service requiring emergency operations were included in this cohort. Trauma patients and EGS patients managed nonoperatively were excluded from this study.

Data were abstracted from the patient's electronic medical records (EMR) and included patient demographic characteristics, diagnosis, type of procedure, comorbidities, length of stay in hospital, 30-day readmission, reoperation, complications, VTE prophylaxis received in hospital, development of clinically suspected VTE, and death. Re-operations included only emergency operations, not planned elective procedures. The EMR covers not only admissions to LHSC, but also presentations to other acute care hospitals in the region. The EMR does not capture visits to primary care physicians. We searched the EMR for 3 months postoperatively for all included patients. Comorbidities were identified from the EMR and quantified by calculating the Charlson Comorbidity Index score. ${ }^{2,3}$ Risk of VTE was quantified using the methodology proposed by Caprini and colleagues ${ }^{4}$ (Appendix 1, available at canjsurg.ca/006318-a1). In the 2012 American College of Chest Physicians (ACCP) Guidelines, patients are categor- ized by Caprini score as very low risk ( $0-1$ points), low risk ( 2 points), moderate risk (3-4 points), and high risk ( $\geq 5$ points). ${ }^{5}$ Complications were classified using the methodology proposed by Clavien and Dindo: grade I represents any deviation from normal postoperative course without the need for pharmacologic treatment; grade II represents a complication requiring pharmacologic treatment; grade III represents a complication requiring surgical, endoscopic, or radiologic intervention; grade IV includes life-threatening complications requiring ICU admission; and grade $\mathrm{V}$ is death. ${ }^{6}$

Clinically suspected VTE was defined a priori as VTE diagnosed with imaging (ultrasonography, computed tomography $[\mathrm{CT}]$, ventilation-perfusion $[\mathrm{VQ}]$ scan) performed in response to signs and symptoms at the discretion of the clinical team. At LHSC, ACS physicians do not perform routine screening for VTE. Appropriate VTE prophylaxis was defined a priori as having chemical prophylaxis ordered for all patients admitted to hospital for more than 48 hours and having patients receive all doses of low molecular weight heparin or unfractionated heparin, with the first dose received within 24 hours of admission. Patients whose hospital stays were shorter than 48 hours were not excluded from the study; in those patients, no VTE prophylaxis was deemed appropriate based on Canadian Patient Safety Institute recommendations from 2015.

\section{Statistical analysis}

Descriptive analysis of patient demographics and clinical characteristics are reported as means \pm standard deviations, medians with interquartile ranges (IQR), and frequencies, as appropriate. Between-group comparisons examine differences in key demographic and clinical characteristics between patients with versus without VTE; those defined as high risk (Caprini $\geq 5$ ) versus low or moderate risk (Caprini 0-4); and those who received appropriate VTE prophylaxis versus those who did not. Betweengroup comparisons were conducted using independent $t$ tests, Mann-Whitney $U$ tests, and $\chi^{2}$ tests, as appropriate $(p<0.05,2$-tailed). All analyses were performed with SPSS software version 22.

\section{Results}

A total of 767 patients were included in this study. The mean age of patients was $53 \pm 20$ years. Fifty-two percent were female, and the median body mass index (BMI) was 27. The median length of stay in hospital was 5 (IQR $1-14)$ days. Eighty-one (10.6\%) patients had active cancer at the time of admission. With respect to risk of VTE, $68.2 \%$ were considered low to moderate risk, while the remaining $31.8 \%$ were considered high risk. The most commonly performed emergency surgeries were appendectomy (23.8\%), cholecystectomy (18.9\%), and large 
$41.7 \%$ of those at low to moderate risk received appropriate VTE prophylaxis. Mortality was significantly higher among those at high risk than among those at low to moderate risk $(17.6 \%$ v. $4.0 \%, p<0.001)$. We performed a subgroup analysis excluding patients with active cancer owing to their known higher risk for VTE to see if this changed the outcomes, ${ }^{8}$ and it did not (Appendix 1, Table S1).

In terms of compliance with VTE prophylaxis at this centre, only $66 \%$ of patients received appropriate VTE prophylaxis (as defined by this study a priori). We compared patients who received appropriate VTE prophylaxis with those who did not (Table 4). The most frequent reason for inappropriate VTE prophylaxis was failure to receive any prophylaxis throughout the duration of their hospital stay (39\%). Twenty-seven percent did not receive their first dose within 24 hours, and another $27 \%$ had missed at least 1 dose during their hospital stay. Four percent of patients refused their VTE prophylaxis, and the remaining 3\% received inappropriate doses (Fig. 1). A significantly higher proportion of patients in the group who received inappropriate VTE prophylaxis underwent reoperation (19.8\% v. 6.3\%, $p=0.002$ ). Of the patients who experience VTEs while in hospital, significantly more patients received inappropriate VTE prophylaxis (4.7\% v. $1.2 \%, p=0.04)$.

\section{Discussion}

In this retrospective cohort study evaluating 767 EGS patients, the overall rate of symptomatic VTE was found

\begin{tabular}{|c|c|c|c|}
\hline Characteristic & $\begin{array}{l}\text { Appropriate } \\
\text { VTE prophylaxis } \\
\quad n=510\end{array}$ & $\begin{array}{c}\text { Inappropriate } \\
\text { VTE } \\
\text { prophylaxis } \\
n=257\end{array}$ & $p$ value \\
\hline Age, yr, mean \pm SD & $50.6 \pm 17.7$ & $57.9 \pm 19.5$ & 0.49 \\
\hline BMI, median [IQR] & $\begin{array}{c}26.7 \\
{[16.8-36.6]}\end{array}$ & $\begin{array}{c}26.8 \\
{[18.7-34.9]}\end{array}$ & 0.45 \\
\hline Female sex, no. (\%) & $277(54.3)$ & $123(47.9)$ & 0.093 \\
\hline $\begin{array}{l}\text { Length of stay, } d \text {, median } \\
\text { [IQR] }\end{array}$ & $6[0-15]$ & $8[0-21]$ & $<0.0001$ \\
\hline Reoperation, no. (\%) & $32(6.3)$ & $51(19.8)$ & 0.002 \\
\hline $\begin{array}{l}\text { 30-day readmission, no. } \\
(\%)\end{array}$ & $27(8.4)$ & $25(10.0)$ & 0.56 \\
\hline $\begin{array}{l}\text { Charlson Comorbidity } \\
\text { Index score, median [IQR] }\end{array}$ & $2[0-7]$ & $3[0-8]$ & 0.14 \\
\hline Caprini score, no. (\%) & & & 0.02 \\
\hline $0-4$ & $378(72.3)$ & $145(27.7)$ & $<0.0001$ \\
\hline$\geq 5$ & $132(54.1)$ & 112 (45.9) & 0.03 \\
\hline Active cancer, no. (\%) & $36(14.0)$ & $45(8.8)$ & 0.034 \\
\hline $\begin{array}{l}\text { VTE during admission, no. } \\
(\%)\end{array}$ & $6(1.2)$ & $12(4.7)$ & 0.04 \\
\hline $\begin{array}{l}\text { VTE after discharge, no. } \\
(\%)\end{array}$ & $6(1.2)$ & $6(2.3)$ & 0.52 \\
\hline Death, no. (\%) & $23(7.2)$ & $28(11.2)$ & 0.11 \\
\hline \multicolumn{4}{|c|}{$\begin{array}{l}\mathrm{BMI}=\text { body mass index; } \mathrm{IQR}=\text { interquartile range; } \mathrm{SD}=\text { standard deviation; } \mathrm{VTE}= \\
\text { venous thromboembolism. }\end{array}$} \\
\hline
\end{tabular}

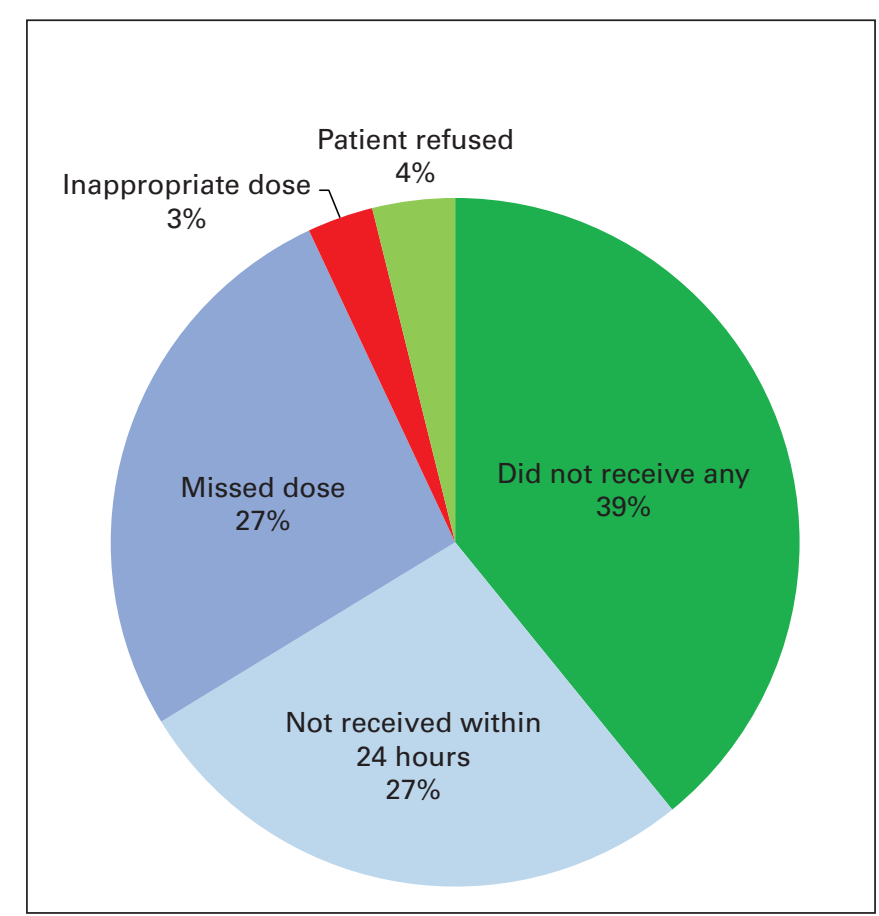

Fig. 1. Reasons for inappropriate venous thromboembolism (VTE) prophylaxis.

to be higher than anticipated, at $3.9 \%$. It is important to note that, in our population, symptomatic VTEs occurred even in patients who were at low risk and in patients who received appropriate VTE prophylaxis. To our knowledge, this study represents one of the first to evaluate VTE in EGS patients specifically and is an important first step in evaluating VTE prophylaxis in this patient cohort.

Venous thromboembolisms are known preventable causes of significant morbidity and mortality. Emergency general surgery patients are in an acutely stressed state from sepsis, inflammation, organ failure, and/or hypovolemia, which increases the risk for VTE. ${ }^{9,10}$ Compared with cancer, trauma, or orthopedic patients, in whom the rate of VTE is $10 \%-20 \%, 2 \%-22 \%$, and $16 \%-30 \%$, respectively, despite VTE prophylaxis, the risk appears to be lower but not insignificant in EGS patients. ${ }^{11-14}$ When compared with patients undergoing elective general surgery procedures, our study shows the rate in EGS patients is higher. A prospective cohort study conducted by Ulrych and colleagues ${ }^{15}$ examined clinically significant DVTs in 216 elective general surgery patients who either underwent elective laparoscopic cholecystectomy or elective hernia repair. Only 1 patient $(0.46 \%)$ experienced DVT in that cohort.

The choice to include VTE diagnosed after hospital discharge allowed us to speculate on the possibilities for extended VTE prophylaxis. Many studies have examined extended prophylaxis for patients at high risk, including surgical cancer patients and orthopedic patients receiving hip or knee replacements. Bergqvist and colleagues ${ }^{8}$ 
conducted a double-blind multicentre trial analyzing whether patients undergoing curative abdominal or pelvic surgery for cancer would benefit from extended VTE prophylaxis of an additional 21 days beyond the initial postoperative period. They concluded that VTE prophylaxis with enoxaparin for 4 weeks after surgery for abdominal or pelvic cancer is safe and significantly reduces incidence of VTE compared with 1-week prophylaxis. The relative risk reduction was $60 \%{ }^{8}$ Extended thromboprophylaxis up to 28 days for high risk general surgery cancer patients and orthopedic hip and knee arthroplasty patients is the current recommendation. ${ }^{5,16}$ However, current recommendations for high risk abdominopelvic surgery patients without cancer are controversial. A Cochrane review and meta-analysis on prolonged thromboprophylaxis for abdominal or pelvic surgery concluded that prolonged thromboprophylaxis with low molecular weight heparin significantly reduces the risk of major VTE; however, there was no significant difference in mortality. ${ }^{17}$ All previous studies used either only cancer patients or both cancer patients and noncancer patients having elective surgery as their study cohorts. Currently, there are no studies that have analyzed whether noncancer, nontrauma, EGS patients requiring major abdominal or pelvic surgery would benefit from extended thromboprophylaxis. Our results show that a significant proportion of VTEs are diagnosed after hospital discharge and suggest that further investigation with regards to extended prophylaxis in EGS patients is warranted.

We also evaluated compliance with appropriate VTE prophylaxis. Slightly more than $60 \%$ of patients received appropriate prophylaxis according to our definition. The 3 top reasons for inappropriate VTE prophylaxis were that patients were not ordered any chemical VTE prophylaxis with a hospital stay longer than 48 hours; patients did not receive the first dose within 24 hours; and patients missed at least 1 dose over the course of their hospital stay. We set a very strict definition for appropriate VTE prophylaxis: receiving dalteparin 5000 units daily or heparin 5000 units twice a day or 3 times a day, with the first dose given within 24 hours of admission, and no missed doses until discharge. We felt a strict definition was valid, given the consequences of even a single missed dose. A prospective study conducted by Louis and colleagues ${ }^{18}$ looked at the rate of DVTs in 202 trauma and general surgery patients to determine whether missing a dose of enoxaparin was associated with higher rate of DVT. They found patients had significantly higher rates of DVT when they missed at least 1 dose of enoxaparin $(23.5 \%$ v. $4.8 \%) .{ }^{18}$ They also identified interrupted enoxaparin therapy as an independent risk factor for DVT formation, demonstrating the importance of not withholding VTE prophylaxis. ${ }^{18}$ Quality-improvement studies on VTE still report poor compliance to appropriate VTE prophylaxis, with com- pliance rates of $58 \%-73 \% .{ }^{19,20}$ Given the findings in our study in which patients experienced VTE despite appropriate prophylaxis, we argue that it is important to have a strict definition of what appropriate VTE prophylaxis is and that centres consider the risk of withholding VTE prophylaxis. Lau and colleagues ${ }^{21}$ conducted a prospective cohort study specifically analyzing compliance to prescribing appropriate VTE prophylaxis by surgical residents and demonstrated that an individualized performance feedback improved the compliance rate from $89.4 \%$ to $95.4 \%(p<0.001)$. An individualized scorecard was sent to all general surgery residents participating in the study (identified by unique study number and not resident name) showing their performance and how they ranked among other residents for 3 months. Not only did this intervention improve resident compliance to prescribing appropriate VTE prophylaxis, it also showed a $52 \%$ relative risk reduction for all VTE and significant reduction of preventable VTEs (0\% v. $0.35 \%, p=0.046) .{ }^{21}$ This was a simple but efective quality-improvement strategy that centres (including our own) should consider implementing to improve their compliance to appropriate VTE prophylaxis.

\section{Limitations}

There are a few limitations in our study. Overall the retrospective nature of the study has inherent selection and information bias. We found that a higher rate of VTE was associated with more frequent hospital readmissions; however, we cannot discern whether VTEs occurred because of hospital readmissions or whether patients were admitted because VTE developed while they were at home. Second, our primary outcome was relatively uncommon (a total of 30 VTEs), limiting the study's power. Third, we assumed that patients in whom V'TE developed were symptomatic, which prompted an imaging investigation and diagnosis. However, given the retrospective nature of this study, we were unable to determine whether some VTEs were diagnosed incidentally. Ideally, asymptomatic patients would be excluded from the VTE group since they might not experience the same morbidity and mortality as symptomatic patients. Fourth, we included all patients undergoing emergency general surgery ranging from laparoscopic appendectomies to exploratory laparotomies. We did not stratify risk of VTE based on initial diagnosis or type of surgery performed, nor did we include nonoperative patients who represent a substantial proportion of EGS patients. Finally, our definition of appropriate VTE prophylaxis did not take weight into consideration. Though controversial, some studies recommend 7500 units of low molecular weighth heparin for patients with morbid obesity, ${ }^{22}$ and this was not considered in this study, which may have led to an overestimation of the rate of appropriate VTE prophylaxis. 


\section{Conclusion}

We identified a $3.9 \%$ rate of VTEs in EGS patients, which is significantly higher than in elective general surgery patients. Our results build upon the limited literature and may be used to inform future guidelines, which, as they currently stand, may not apply to EGS patients. Further research is warranted to identify reasons why patients are not receiving appropriate VTE prophylaxis, and steps should be taken to improve compliance. Finally, further investigations into whether high risk EGS patients warrant prolonged prophylaxis should be conducted.

Affiliations: From the Division of General Surgery, Department of Surgery, Schulich School of Medicine and Dentistry, Western University, London, Ont. (Yang, Murphy, Sela, Govind, Leslie, Vogt); and the London Health Sciences Centre, London, Ont. (Allen).

Competing interests: K. Vogt is an associate editor of CFS. She was not involved in the review of this manuscript or in the decision to accept it for publication. No other competing interests declared.

Contributors: K. Leslie and K. Vogt designed the study. M. Yang, N. Sela and S. Govind acquired the data, which M. Yang, L. Allen and K. Vogt analyzed. M. Yang and K. Vogt wrote the article, which all authors reviewed and approved for publication.

\section{References}

1. Buesing KL, Mullapudi B, Flowers KA. Deep venous thrombosis and venous thromboembolism prophylaxis. Surg Clin N Am 2015;285-300.

2. Charlson ME, Pompei P, Ales KL, et al. A new method of classifying prognostic comorbidity in longitudinal studies: development and validation. 7 Chronic Dis 1987;40:373-83.

3. Quan H, Sundararajan V, Halfon P, et al. Coding algorithms for defining comorbidities in ICD-9-CM and ICD-10 administrative data. Med Care 2005;43:1130-9.

4. Caprini JA, Arcelus JI, Hasty JH, et al. Clinical assessment of venous thromboembolic risk in surgical patients. Semin Thromb Hemost 1991;17:304-12.

5. Gould MK, Garcia DA, Wren SM, et al. American College of Chest Physicians. Prevention of VTE in nonorthopedic surgical patients. Chest 2012;141(Suppl):e227S-77S.

6. Dindo D, Demartines N, Clavien PA. Classification of surgical complications. Ann Surg 2004;240:205-13.

7. Geerts W, Diamantouros A. Venous thromboembolism prevention — getting started kit. Ottawa: Canadian Patient Safety Institute; 2019. Available: https://www.patientsafetyinstitute.ca/en/toolsResources/
Documents/Venous\%20Thromboembolism\%20(VTE)/English\%20 PDFs/VTE\%20GSK\%20EN.pdf (accessed 2019 June 11).

8. Bergqvist D, Agnelli G, Cohen AT, et al. Duration of prophylaxis against venous thromboembolism with enoxaparin after surgery for cancer. NEngl 7 Med 2002;346:975-80.

9. Saghazadeh A, Rezae N. Inflammation as a cause of venous thromboembolism. Crit Rev Oncol Hematol 2016;99:272-85.

10. Kaplan D, Casper TC, Elliott CG, et al. VTE incidence and risk factors in patients with severe sepsis and septic shock. Chest 2015;148:1224-30.

11. Rasmussen MS. Preventing thromboembolic complications in cancer patients after surgery: A role for prolonged thromboprophylaxis. Cancer Treat Rev 2002;28:141-4.

12. Geerts WH, Jay RM, Code KI, et al. A comparison of low-dose heparin with low-molecular weight heparin as prophylaxis against venous thromboembolism after major trauma. NEngl F Med 2002;335:701-7.

13. O'Malley KF, Ross SE. Pulmonary embolism in major trauma patients. F Trauma 1990;30:748-50.

14. Douketis JD, Wikelboom JW, Quinlan DJ, et al. Short-duration prophylaxis against venous thromboembolism after total hip or knee replacement. Arch Intern Med 2002;162:1465-71.

15. Ulrych J, Kvasnicka T, Fryba V, et al. 28 day post-operative persisted hypercoagulability after surgery for benign diseases: a prospective cohort study. BMC Surg 2016;16:1-8.

16. Falck-Ytter Y, Francis CW, Johanson NA, et al. American College of Chest Physicians. Prevention of VTE in orthopedic surgery patients. Chest 2012;141(Suppl):e278S-325S.

17. Rasmussen MS, Jorgensen LN, Wille-Jorgensen P. Prolonged thromboprophylaxis with low molecular weight heparin for abdominal or pelvic surgery [review]. Cochrane Database Syst Rev 2009;1:1-19.

18. Louis SG, Sato M, Geraci T, et al. Correlation of missed doses of enoxaparin with increased incidence of deep vein thrombosis in trauma and general surgery patients. FAMA Surg 2014; 149:365-70.

19. Cohen AT, Tapson VF, Bergmann JF, et al. Venous thromboembolism risk and prophylaxis in the acute hospital care setting (ENDORSE study): a multinational cross-sectional study. Lancet 2008;371:387-94.

20. Geahchan N, Basile M, Tohmeh M. Venous thromboembolism prophylaxis in patients undergoing abdominal and pelvic cancer surgery: adherence and compliance to ACCP guidelines in DIONYS registry. Springerplus 2016;5:1541.

21. Lau BD, Arnaoutakis GJ, Streiff MB, et al. Individualized performance feedback to surgical residents improves appropriate venous thromboembolism prophylaxis prescription and reduces potentially preventable VTE. Ann Surg 2015;264:1181-7.

22. Vandiver J, Ritz L, Lalama J. Chemical prophylaxis to prevent venous thromboembolism in morbid obesity: literature review and dosing recommendations. 7 Thromb Thrombolysis 2016;41:475-81. 